\title{
MAPPING OF ARBITRARY TRAFFIC DEMAND AND NETWORK TOPOLOGY ON A MESH OF RINGS NETWORK
}

\author{
Christian Mauz \\ Communication Technology Laboratory \\ ETH Zurich \\ Switzerland \\ mauz@nari.ee.ethz.ch
}

\begin{abstract}
An efficient algorithm is presented to map an arbitrary traffic pattern and network topology on a mesh of rings network. The network is described by a two-layer model. The first layer defines the ring configuration and the second the connection allocation. Rings are constructed systematically from faces. It is assumed that a ring provides a fullmeshed network with a certain capacity between every node pair. Simulated annealing (SA) is used to optimize the mapping to best fit the traffic demands. For that, an objective function with certain parameters is introduced. It is possible to incorporate boundary conditions, e.g. the maximum number of nodes in a ring in this function. The algorithm is tested on the basis of the COST 239 Pan-European case study as an example.
\end{abstract}

\section{INTRODUCTION}

Wavelength-division multiplexing is used to an increasing extent for point-to-point connections in wide area networks to satisfy the demand for transport capacity. SDH (Synchronous Digital Hierarchy) or SONET (Synchronous Optical Network) represent the classic transport protocol.

Two approaches can be applied to design a communication network. The first one is connection-based i.e. the routing will be computed for every connection. The second one is structure-based: the network will be built up from blocks like rings or meshes. Several architectures based on regular topologies for all-optical networks e.g. the Colored Section Ring [Ham96] or meshed topologies e.g. the Gridconnect [Jag99], have been proposed and investigated. These concepts assume a certain topology and a homogeneous traffic matrix. The mapping of the real-world 
topologies and the traffic demand on such simple networks is a challenging task due to the many degrees of freedom. H. Jäger proposed a mapping for the Gridconnect which has the disadvantage of high computational complexity [Jag96].

The aim of this work is to propose an efficient semi-heuristic algorithm with rather low complexity for the mapping of arbitrary topology and traffic pattern on a mesh of rings. It is possible to set in advance a certain fiber topology as well as to compute an optimum one. To justify this approach, an implementation of the algorithm using the C++ library LEDA [Meh96] has been made and the "Pan-European Network" of the COST 239 project has been used as a test-scenario.

To clarify the presentation, not a certain realization of a ring is considered. The ring network is treated as a black box which provide a full-meshed network with a certain transport capacity between the node pairs. For the same reason, protection has been neglected even though this is the main reason to introduce rings.

The structure of the paper is as following. First a two layer graph model is introduced. The ring layer and a systematic way to construct rings out of smaller pieces is presented. Then, the resulting effective graph and the routing thereon is explained. For the optimization "Simulated Annealing" (SA) is used. The results for the Pan-European Network are analyzed. The short summary at the end includes an outlook for further work.

\section{MULTI-LAYER GRAPH MODEL}

For the description of the network, a two-layer graph model is used (see Fig. 1). The lower layer contains the real network nodes and the real fiber topology. The actual configuration is a set of rings: each ring consists of a set of nodes and edges of the network.

The upper layer is the connection layer. It describes the effective, logical topology which results from the set of rings of the lower layer. Here, for every connection request of the traffic matrix, a path through this logical network has to be found.

The general aim is to minimize the ring configuration with respect to a given objective function so that all traffic demands are fulfilled and the boundary conditions, e.g. the capacity per link, are not violated.

In this work, only the ring identification and the routing in the logical network is investigated. It is assumed, that for the realization of one of these rings, an efficient solution is known (e.g. the Colored Section Ring or an SDH ring). Therefore, for example, the mapping of the resulting channels to distinct wavelengths is not treated. This could be easily 

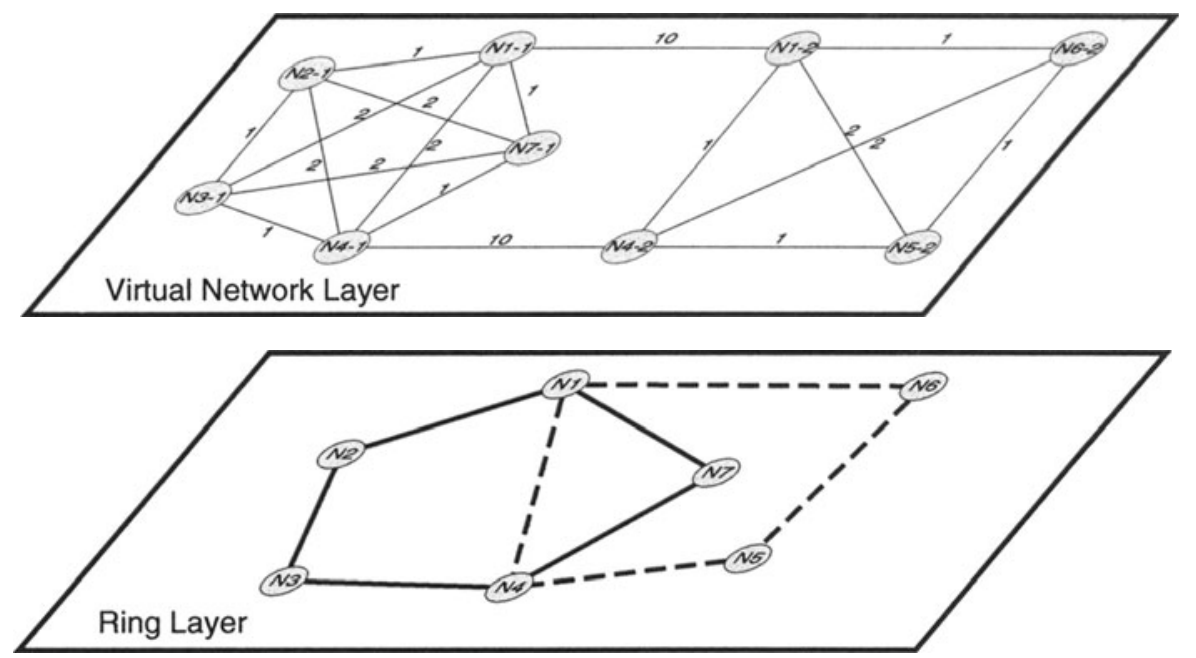

Figure 1 Two layer graph model. The lower layer shows a two ring configuration (solid and dashed edges). In the virtual network layer, the resulting effective graph is depicted. The edges are labeled with the distances. N1-1 and N1-2 are the two virtual copies of $\mathrm{N} 1$.

integrated in the algorithm by adding a third layer and using one of the known algorithms for wavelength allocation e.g. "First-Fit" [Har98].

\section{THE RING-LAYER}

A map is a graph for which the reversal information for every edge is defined. An embedding of a graph is a drawing, where no edge is drawn across a node, images of distinct edges do not cross, and multiple edges between two nodes are embedded the same. A map for which an embedding in the plane exists, is a planar map. A famous theorem of Kuratowski states [Kur30], that every map can be decomposed into a planar map and Kuratowski sub-graphs $K_{5}$ (complete graph of degree 5 ) and $K_{3,3}$ (complete bipartite graph with $2 \times 3$ nodes). For the two sub-graphs, a realization by an all-optical network is straight-forward. In addition, due to the high connectivity of these graphs, they will normally not be found in transport networks. Therefore it is sufficient to assume a planar map in the following. Details for graph theory may be found in [Whi73].

In graph theory, there exists the combinatorial and geometrical concept of a face. A face is a list of edges. To construct a face, one starts at 

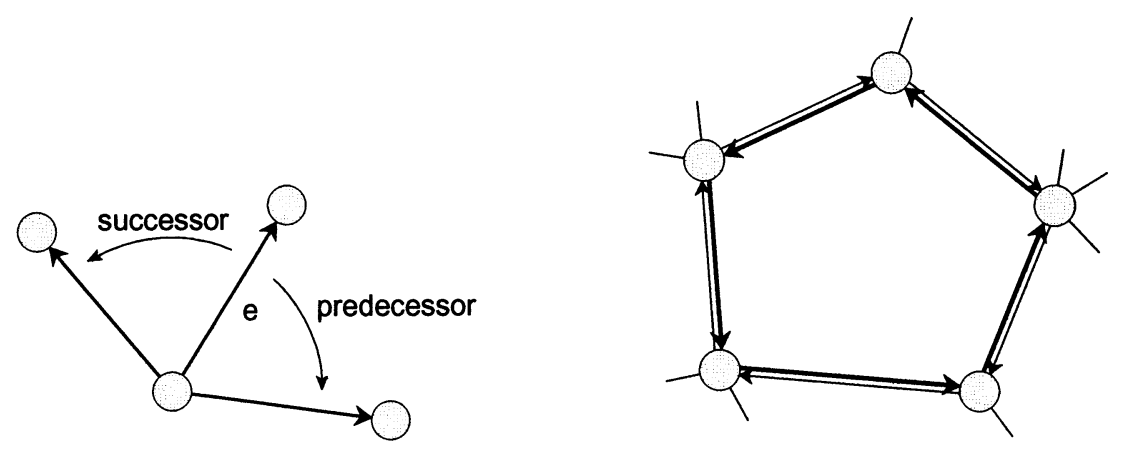

Figure 2 On the left side the definition of the successor and predecessor of the edge $e$ is displayed. On the right side, the constructed face and its edges are marked with thick lines.

a node, follows the edges, and takes at every node the preceding outgoing edge to the next node until one returns to the starting node (see Fig. 2). It is possible to decompose every planar map into faces with complexity linear to the number of edges in the graph.

These faces are the building blocks for rings and form a basis for the space of cycles in a graph. A face is the smallest possible ring in a network. By the addition of a neighbor face to a ring, the ring may be enlarged. On the other hand, to shrink the ring, one of the faces which share an edge with the border of the ring has to be removed. It is worth to note, that this operation is self-inverse: addition of two times the same face to a ring does not change the ring. Some cautions has to be taken for both operations. In some special cases (see an example in Fig. 3), the result is no longer a ring. By using the face concept, it is possible to construct rings in a systematic way with low complexity. Every ring is described by a set of faces. The removal of the last face of a ring destroys the ring. On the other side, the addition of one face to an empty set creates a new ring.

One configuration of the ring layer stands for a set of rings or in other words a mesh of rings with distributed interfaces.

For a given node configuration and edge topology, all faces may be computed. On the other hand, it is possible to use a triangulation algorithm of the plane for the generation of a triangular mesh when only the nodes and their positions are given to gèt a starting topology. At the end, some of these links may not be used. 


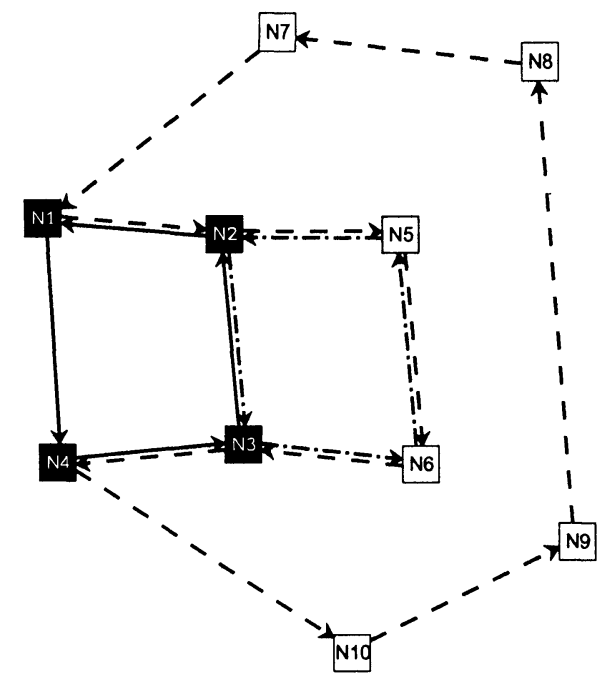

Figure 3 Problem which occurs when enlarging a ring (solid line and black nodes) by a face. The dashed face can't be added to the ring because the result would not be a ring, only the addition of the dot-dashed face is possible.

\section{THE VIRTUAL NETWORK LAYER}

The ring configuration provides an effective network topology for the connections. Every ring in the lower layer is translated into a complete graph of the virtual network layer. In a complete graph a direct link exists between every node. Nodes which belong to several rings, create multiple copies in the effective graph, where all copies are connected by a bridging link.

For the simulation it is assumed that every ring link has a capacity of four connections. This is e.g. the case for a traffic matrix with granularity of STM-16 $(2.5 \mathrm{Gbit} / \mathrm{s})$ and a ring technology which offers STM-64 (10 Gbit/s) channels. The connections could be multiplexed using SDH/SONET technology. The bridging links are not limited in the number of connections they can carry.

For the routing, it is necessary to assign a distance to every link. Here, the inter-ring distance is the number of nodes which are passed in the real network topology and the distance for the bridging links is choosen as $d_{\text {bridge }}=10$ in the examples. This takes into account that changing the ring results in a demultiplexing and a multiplexing operation, which is rather expensive. In general, the value of $d_{\text {bridge }}$ depends on the ratio between the cost for bandwith and the cost for multiplexing or demultiplexing, and may even be a function of the network size. 
In Fig. 1 an example for a ring-configuration and the resulting effective graph in the higher layer is shown.

For every connection request of the traffic matrix, a connection has to be assigned on the effective graph. Since there is the abstraction of the concrete realization of one ring, this is trivial for connections which can be handled within a direct channel of the ring. For the remaining ones (in the following referred to as "hop connections"), a minimization of the path-length is aspired. Possible paths, e.g. the ten shortest paths, through the network for every node pair are computed with one of the standard algorithms [Had99]. In case that the start or the end node belong to several rings, all virtual copies of it are treated in the same way.

The hop connection requests are assigned in random order. For every request, starting with the shortest possible path, it is checked if enough capacity remains on all links. If this is the case, a solution has been found, otherwise the next longer possible path is examined (in the simulation at least $n=10$ paths are investigated). When there is no possible routing found among the computed possible paths, the connection is added to a special list of not-fulfilled connections. It would be possible to route this traffic manually in the standard connection-oriented style.

Due to the fact that the granularity of the ring connections is higher than that of the traffic matrix, also the grooming of the different connections can be solved.

For simplicity, protection against link or node failure has not been investigated. Though it is straightforward to take a combination of working and protection paths to provide $1+1$ protection, instead of handling only one path between the node pairs. This is solely important for the connections passing through several rings because normally, the ring management will provide inter-ring protection.

\section{THE OPTIMIZATION PROCESS}

Due to the high degrees of freedom in network optimization problems, it is impossible to compute all configurations to find the optimum solution. Therefore one has to rely on approximation methods. One very well-known method which has proved in this class of problems is "Simulated Annealing". Any other known optimization method like tabusearch or genetic optimization, which are not trapped in local minima, could be used as well.

As a starting configuration, all faces of the graph are computed and assigned as a ring to the set of rings. The translation in an effective 
network graph and the routing on this network is done as described above.

In every step, a neighbor configuration is derived from the actual configuration. One of the following operations is chosen at random to derive the neighbor configuration:

- adding one face to one of the rings,

- creating a new ring with a random face,

- deleting a face of a ring (when there are no faces left in the set of faces of the ring, the ring is destroyed),

- change of the order in which the hop connections are assigned.

For the optimization process the evaluation of the configuration is of crucial importance. Here the sum of the length of all connections is used. For every connection which could not be routed, a penalty of $p_{n p}$ is added

$$
C_{\mathrm{cfg}}=\sum_{p \in R_{p}} d(p)+\sum_{n p \in R_{\mathrm{np}}} p_{n p},
$$

where $C_{\text {cfg }}$ denotes the cost of the configuration, $R_{\mathrm{p}}$ and $R_{\mathrm{np}}$ are the sets of routed paths and the node-pairs for which no connection has been found, respectively. For the example, $p_{n p}=1000$ has been choosen. The distance for the path $p$ is devoted as $d(p)$. It is simple to incorporate any other design criteria, e.g. the maximum diameter or the maximum number of nodes for a ring.

The objective function for the actual configuration and the neighbor configuration are compared. When the cost for the neighbor configuration $C_{\text {ncfg }}$ is lower than for the actual configuration $C_{\text {cfg }}$, it is accepted in any case. Otherwise the worse neighbor configuration is accepted for

$$
r<\exp \left(-\frac{C_{\mathrm{ncfg}}-C_{\mathrm{cfg}}}{T}\right)
$$

with $r \in[0,1]$ a random number with uniform distribution. This prevents to be trapped in a local minima of the objective function. $T$ is a state variable of the optimization process which is referred to as the temperature. At the beginning, $T$ is chosen such that more or less every configuration is accepted. It is lowered in the following steps for that at the end, only better configuration with lower cost are accepted. Here, for $T$ an exponential cooling has been chosen.

One process of SA is used for changes of the routing and topology. To improve the results, a two-step way may be favorable where one SA process optimizes the ring-configuration and a second SA tries to minimize the resulting virtual network. 


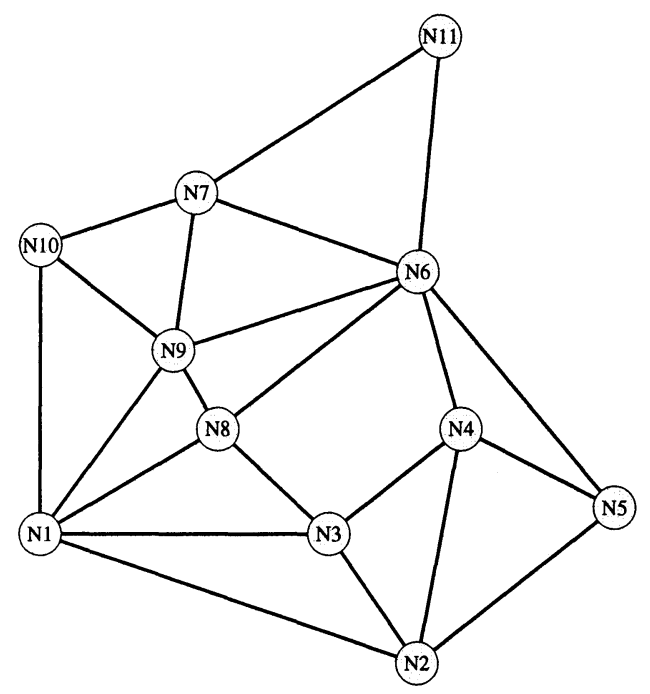

Figure 4 Fiber topology according to the COST 239 case study for a Pan-European Network.

\section{A CASE STUDY FOR THE "PAN-EUROPEAN NETWORK" OF THE COST 239 PROJECT}

In the framework of the European project COST 239, several case studies for a Pan-European network have been performed [Bat00]. In Fig. 4 the given fiber topology and in Tab. 1 the corresponding traffic matrix are depicted. For the example, a granularity of $2.5 \mathrm{Gbit} / \mathrm{s}$ per connection has been assumed. The presented algorithm has been implemented in C++ using the library for discrete math and graphs LEDA and tested using the COST 239 scenario as a test example. All parameters of the objective function (distances, capacities, penalties) has been choosen as descripted before. In total 348 connections have to be established.

A typical example for the resulting ring configuration is

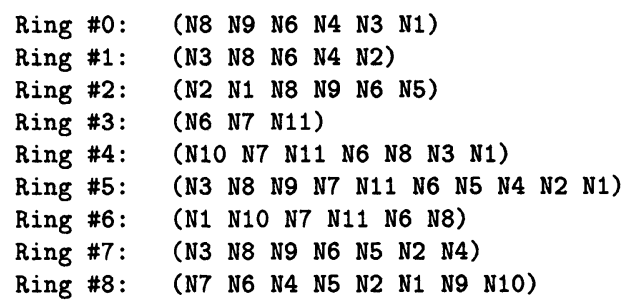




\begin{tabular}{|c|c|c|c|c|c|c|c|c|c|c|c|}
\hline & N1 & N2 & N3 & N4 & N5 & N6 & N7 & N8 & N9 & N10 & N11 \\
\hline \hline N1 & 0 & 12.5 & 15 & 2.5 & 5 & 27.5 & 12.5 & 2.5 & 17.5 & 25 & 2.5 \\
\hline N2 & 12.5 & 0 & 15 & 2.5 & 7.5 & 22.5 & 5 & 2.5 & 5 & 7.5 & 2.5 \\
\hline N3 & 15 & 15 & 0 & 2.5 & 7.5 & 27.5 & 7.5 & 2.5 & 7.5 & 7.5 & 2.5 \\
\hline N4 & 2.5 & 2.5 & 2.5 & 0 & 2.5 & 5 & 2.5 & 2.5 & 2.5 & 2.5 & 2.5 \\
\hline N5 & 5 & 7.5 & 7.5 & 2.5 & 0 & 22.5 & 2.5 & 2.5 & 2.5 & 5 & 2.5 \\
\hline N6 & 27.5 & 22.5 & 27.5 & 5 & 22.5 & 0 & 20 & 5 & 15 & 20 & 7.5 \\
\hline N7 & 12.5 & 5 & 7.5 & 2.5 & 2.5 & 20 & 0 & 2.5 & 10 & 12.5 & 2.5 \\
\hline N8 & 2.5 & 2.5 & 2.5 & 2.5 & 2.5 & 5 & 2.5 & 0 & 2.5 & 2.5 & 2.5 \\
\hline N9 & 15 & 5 & 15 & 2.5 & 2.5 & 15 & 10 & 2.5 & 0 & 10 & 2.5 \\
\hline N10 & 25 & 7.5 & 7.5 & 2.5 & 5 & 20 & 12.5 & 2.5 & 10 & 0 & 2.5 \\
\hline N11 & 2.5 & 2.5 & 2.5 & 2.5 & 2.5 & 7.5 & 2.5 & 2.5 & 2.5 & 2.5 & 0 \\
\hline
\end{tabular}

Table 1 Traffic matrix for the COST 239 case study for a Pan-European Network in units of $\mathrm{Gbit} / \mathrm{s}$.

\section{Optimization process: temperature and costs}

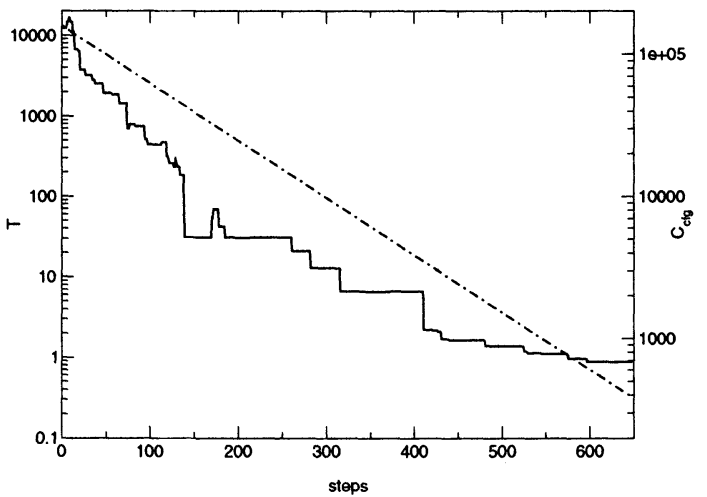

Figure 5 Trend of temperature and cost function during the optimization process without restriction on ring size. Dashed line and right axis belongs to the temperature $T$, solid line and left axis belongs to the configuration costs $C_{\mathrm{cfg}}$.

In Fig. 5 the corresponding course of the cooling and cost is depicted. At the beginning 131 connection requests can not be established. After 410 steps, for every demand a connection can be allocated. The final configuration with 9 rings has a total cost of 683 and has evolved after 647 steps. Despite the fact that the implementation is not optimized (e.g. in every step a complete routing and not only the necessary changes due to a change in topology or other possible routings for every node pair are calculated even if the specific connection can be handled by a ring), the running time on a Pentium 500 computer is below one hour. 
Optimization process: temperature and costs

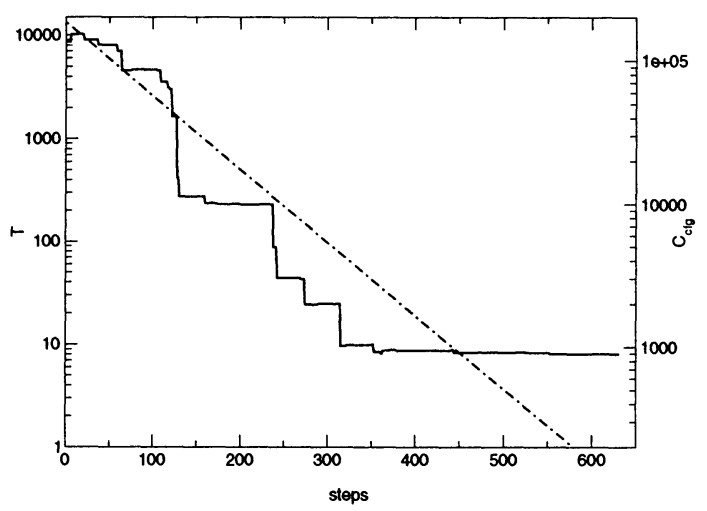

Figure 6 Trend of temperature and cost function during the optimization process with restriction on ring size.

All connections are handled by rings and no hop connections are necessary. It is worth to note, that no restriction on the ring size has been imposed. For the example this results in rather large rings with up to 10 nodes which are difficult to realize. Therefore, in a second run a limit in the ring size of 8 nodes is set by adding a penalty of 1000 to larger rings. The result for the changed objective is

$\begin{array}{ll}\text { Ring \#0: } & \text { (N7 N11 N6 N9 N10) } \\ \text { Ring \#1: } & \text { (N4 N3 N1 N8 N9 N6 N5) } \\ \text { Ring \#2: } & \text { (N9 N7 N6 N4 N3 N8 N1) } \\ \text { Ring \#3: } & \text { (N8 N3 N4 N2 N1 N10 N9 N6) } \\ \text { Ring \#4: } & \text { (N8 N6 N5 N2 N3 N1 N10 N9) }\end{array}$

In Fig. 6 the corresponding trend for cost function and temperature is depicted. Now, in total 5 rings are necessary to fulfill all traffic demands. The final configuration with a cost of 898 has evolved after 630 steps. Thus, 30 hop connections are necessary due to the smaller size of the rings. Therefore, the cost of the solution is slightly higher than the previous one without that boundary condition.

\section{CONCLUSIONS AND OUTLOOK}

A two-layer graph model with a ring layer and an effective network layer has been presented. Using faces as building blocks allows a systematic and efficient description of the ring configuration. The allocation of the connection for the actual traffic demands takes place on the resulting effective network. SA is used to optimize the ring configuration with respect to an objective function. Boundary conditions can be incorporated 
in this function. Therefore, it is possible to map an arbitrary network topology and traffic demand on a mesh of rings network. The algorithm has been tested with the COST 239 Pan European Case study. Only 300-700 steps are necessary to find a good realization for this network.

There are several possibilities to improve the efficiency of the implementation, especially with respect to the routing in the effective network layer. Also, an interleaved optimization of the ring configuration and the routing on top of it with two separate SA would be favorable.

A specific realization of the rings has not been treated until now. The method can be easily extended to incorporate a third layer for the wavelength paths. This would enable, e.g. an optimization with respect to the number of necessary wavelengths or the treatment of the wavelength allocation problem.

\section{Acknowledgments}

The author is grateful to Dr. J. Kemper for the carefully reading of the manuscript and his various suggestions and to the reviewers helping to clarify the presentation. The author would also like to thank Prof. P. Leuthold, Director of the Communication Technology Laboratory, ETH Zürich, for his support. This work has been performed in the framework of the COST 266 action which was partly founded by the Swiss BBW (Bundesamt für Bildung und Wissenschaft).

\section{References}

[Ham96] A. Hamel et al, "Increased Capacity in an MS Protection Ring using WDM Technique and OADM: The Coloured Section Ring", IEE Electr. Lett., Vol. 32, p. 234-235, Feb. 1996

[Jag99] H.A. Jäger, "The Modular WDM-Gridconnect as a Passive Routing Structure with Distributed Interfacing Capabilities", Photon. Netw. Comm., Vol. 1, No. 1, p. 23-34, June 1999

[Jag96] H.A. Jäger and T. Gipser, "Mapping of Virtual Regular Mesh Network Topologies onto Arbitrary Geographical Node Distributions", Proc. of Conf. on Netw. and Opt. Comm. (NOC), ISBN 905199 274, Vol. 3, p. 64-71, June 1996

[Har98] H. Harai, M. Murata und H. Miyahara, "Performance Analysis of Wavelength Assignment Policies in All-Optical Networks with Limited-Range Wavelength Conversion", IEEE J. on Selected Areas in Comm., Vol. 16, No. 7, p. 1051-60, Sept. 1998

[Kur30] C. Kuratowski, "Sur le problème the courbes guaches en topologie", Fundamenta Mathematicae, Vol. 15, p. 271-83, 1930 
[Meh96] K. Mehlhorn and S. Näher, "LEDA, A Platform for Combinatorial and Geometric Computing", Cambridge University Press, 1996, http://www.mpi-sb.mpg.de/LEDA/leda.html

[Whi73] A.T. White, "Graphs, Groups, and Surfaces", North Holland, 1984

[Had99] E. Hadjiconstantinou and N. Christofides, "An efficient implementation of an algorithm for finding K shortest simple paths", Networks, vol. 34, no. 2, p.88-101, Sept. 1999

[Bat00] P. Batchelor et al, "Study on the implementation of optical transparent transport networks in the European environment-results of the research project COST 239", Photon. Netw. Comm., Vol. 2, No. 1, p.15-32, Jan.-March 2000 